\title{
Activation of Aryl Halides by Nickel(I) Pincer Complexes: Reaction Pathways of Stoichiometric and Catalytic Dehalogenations
}

Christoph A. Rettenmeier, Jan Wenz, Hubert Wadepohl and Lutz H. Gade

Supporting Information

S1. Experimental Procedures

S1.1. General Procedures

S1.2 Preparation of Compounds

S1.3 Additional Experiments

S2. Computational Data

S3. Crystallographic Data

Table S1. Details of the crystal structure determinations 


\section{S1. Experimental Procedures}

\section{S1.1. General Procedures}

All manipulations of air- and moisture-sensitive materials were carried out under an inert atmosphere of dry argon (Argon 5.0 purchased from Messer Group $\mathrm{GmbH}$ and dried over Granusic ${ }^{\circ}$ phosphorpentoxide granulate) using standard Schlenk techniques or by working in a glove box. The solvents were dried over sodium (toluene), potassium (hexane) or sodium/potassium alloy (pentane, diethyl ether), distilled and degassed prior to their use. ${ }^{1}$ Deuterated solvents were purchased from Deutero $\mathrm{GmbH}$ and or from Euriso-Top $\mathrm{GmbH}$, dried over potassium $\left(\mathrm{C}_{6} \mathrm{D}_{6}\right.$, toluene-d8, thf-d8), vacuum distilled, degassed and stored in teflon valve ampoules under argon. Hydrogen 5.0 (Messer Group $\mathrm{GmbH}$ ) was used as purchased without further purification. The phenyl-substituted 2,5bis(oxazolinylmethyl)pyrrole protioligands ${ }^{2}$ as well as the complexes $\operatorname{LigNi}^{\mathbf{I}}(\mathbf{1}),{ }^{3} \mathbf{L i g N i C l}(\mathbf{2})^{3}$ and LigNiBr $(3)^{3}$ were synthesized according to literature procedures. All other reagents were obtained from commercial sources and were used as received unless explicitly stated otherwise.

Air-sensitive samples for NMR spectroscopy were prepared under argon in $5 \mathrm{~mm}$ Wilmad tubes equipped with J. Young Teflon valves. ${ }^{1} \mathrm{H}$ - and ${ }^{13} \mathrm{C}$-NMR spectra were recorded on a Bruker Avance (200 MHz), a Bruker Avance II (400 MHz) and a Bruker Avance III (600 MHz, equipped with a CryoProbe ${ }^{\mathrm{TM}}$ ) NMR spectrometers and were referenced internally using the residual protio solvent $\left({ }^{1} \mathrm{H}\right)$ or solvent $\left({ }^{13} \mathrm{C}\right) .{ }^{4}$ The appearance of the signals was described using the following abbreviations: $\mathrm{s}$ (singlet), d (doublet), dd (doublet of doublet), ddd (doublet of doublet of doublet), dt (doublet of triplet), $\mathrm{t}$ (triplet), q (quartet), $\mathrm{m}$ (multiplet), b (broad signal).

Continuous-wave X-band (ca. $9 \mathrm{GHz}$ ) EPR spectra were acquired using a Bruker Biospin Elexsys E500 EPR spectrometer fitted with a super high Q cavity. The magnetic field and the microwave frequency were calibrated with a Bruker ER 041XK Teslameter and a Bruker microwave frequency counter. The temperature of the sample was adjusted using a flow-through cryostat in conjunction with a Eurotherm (B-VT-2000) variable-temperature controller. EPR spectra simulations were carried out using the XSophe software (Bruker, version 1.1.4).

Elemental analyses were recorded by the analytical service of the Heidelberg Chemistry Department using the vario EL and vario MIKRO cube analytical devices.

Mass spectra were acquired on Bruker ApexQe hybrid 9.4 T FT-IVR (HR-ESI, HR-DART) and JEOL JMS-700 magnetic sector (HR-FAB, HR-EI, LIFDI) spectrometers at the mass spectrometry facility of the Organic Department at the University of Heidelberg. Either 3-nitrobenzyl alcohol (NBA) or $o$ nitrophenyloctyl ether (NPOE) were used as matrix in the FAB-MS measurements.

X-Ray diffraction analysis was performed at the laboratory for structural analysis of the Inorganic Chemistry Department at the University of Heidelberg under the supervision of Prof. Dr. Wadepohl. An Agilent Technologies Supernova-E CCD $\left(\mathrm{Cu}-\mathrm{K}_{\alpha}\right.$ or $\mathrm{Mo}-\mathrm{K}_{\alpha}$ X-radiation, microfocus tube, multilayer mirror optics) and a Bruker AXS Smart 1000 CCD diffractometer (Mo $\left(\mathrm{K}_{\alpha}\right)$ radiation, graphite monochromator, $\lambda=0.71073 \AA$ ) was used for data acquisition.

UV/Vis spectra were recorded on a Varian Cary 5000 UV/VIS/NIR spectrometer. 


\section{S1.2. Preparation of Compounds}

\section{Preparation of complex $\operatorname{LigNiC}_{6} \mathrm{~F}_{5}\left(4-\mathrm{F}_{5}\right)$}

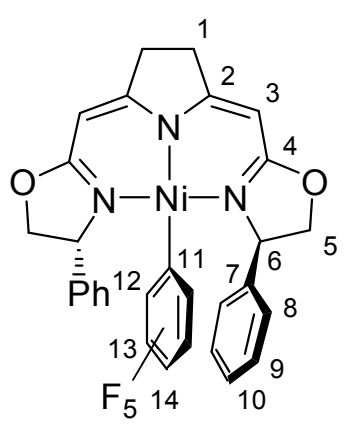

A solution of the bromido complex 3 (69 $\mathrm{mg}, 0.13 \mathrm{mmol}$ ), chloropentafluorobenzene $(0.70 \mathrm{mmol}, 5.3 \mathrm{eq})$ and magnesium $(0.82 \mathrm{mmol}$, $6.2 \mathrm{eq})$ in $5 \mathrm{ml}$ THF was stirred at room temperature for $30 \mathrm{~min}$. After removal of the solvents, a toluene/pentane mixture (1/15) was added, the reaction mixture was stirred for $4 \mathrm{~h}$ and subsequently filtrated. After removal of the solvents, the crude was recrystallized from a THF/pentane mixture at $-40{ }^{\circ} \mathrm{C}$ to give a yellow crystalline solid in $84 \%$ yield $(68 \mathrm{mg})$. The synthesis of 4-F $\mathbf{F}_{5}$ was also performed using previously prepared Grignard reagent $\mathrm{BrMgC}_{6} \mathrm{~F}_{5}$ in THF instead of the one-pot methodology described above.

\section{${ }^{1} \mathrm{H}-\mathrm{NMR}\left(\mathrm{C}_{6} \mathrm{D}_{6}, 600.130 \mathrm{MHz}, 295 \mathrm{~K}\right)$ :}

$\delta(\mathrm{ppm})=7.10\left(\mathrm{~m}, 4 \mathrm{H}, \mathrm{H}^{9}\right), 7.05\left(\mathrm{~m}, 2 \mathrm{H}, \mathrm{H}^{10}\right), 6.65\left(\mathrm{~m}, 4 \mathrm{H}, \mathrm{H}^{8}\right), 5.03\left(\mathrm{~s}, 2 \mathrm{H}, \mathrm{H}^{3}\right), 3.70\left(\mathrm{dd},{ }^{3} J=9.0\right.$ $\left.\mathrm{Hz},{ }^{3} J=3.0 \mathrm{~Hz}, 2 \mathrm{H}, \mathrm{H}^{6}\right), 3.45\left(\mathrm{dd},{ }^{2} J=8.3 \mathrm{~Hz},{ }^{3} J=8.5 \mathrm{~Hz}, 2 \mathrm{H}, \mathrm{H}^{5}\right), 3.45\left(\mathrm{dd},{ }^{2} J=8.3 \mathrm{~Hz},{ }^{3} J=3.0\right.$ $\left.\mathrm{Hz}, 2 \mathrm{H}, \mathrm{H}^{5^{\circ}}\right), 2.14-2.00\left(\mathrm{~m}, 4 \mathrm{H}, \mathrm{H}^{1,1^{\circ}}\right)$.

\section{${ }^{13} \mathrm{C}-\mathrm{NMR}\left(\mathrm{C}_{6} \mathrm{D}_{6}, 150.903 \mathrm{MHz}, 295 \mathrm{~K}\right)$ :}

$\delta(\mathrm{ppm})=172.2 \quad\left(\mathrm{C}^{2}\right), \quad 166.0 \quad\left(\mathrm{C}^{4}\right), \quad 149.1 \quad\left(\mathrm{C}^{12 / 13 / 14}{ }_{,} J_{\mathrm{CF}}=231.3 \mathrm{~Hz}\right), \quad 142.9 \quad\left(\mathrm{C}^{7}\right), \quad 138.4$ $\left(\mathrm{C}^{12 / 13 / 14}{ }_{1}^{1} J_{\mathrm{CF}}=231.3 \mathrm{~Hz}\right), 135.3\left(\mathrm{C}^{12 / 13 / 14}{ }^{l} J_{\mathrm{CF}}=231.3 \mathrm{~Hz}\right), 128.8\left(\mathrm{C}^{9}\right), 127.9\left(\mathrm{C}^{10}\right), 125.2\left(\mathrm{C}^{8}\right), 116.1$ $\left(\mathrm{C}^{11}\right), 81.6\left(\mathrm{C}^{3}\right), 73.4\left(\mathrm{C}^{5}\right), 69.9\left(\mathrm{C}^{6}\right), 30.7\left(\mathrm{C}^{1}\right)$.

${ }^{19} \mathrm{~F}-\mathrm{NMR}\left(\mathrm{C}_{6} \mathrm{D}_{6}, 376.273 \mathrm{MHz}, 295 \mathrm{~K}\right)$ :

$\delta(\mathrm{ppm})=-116.6\left(\mathrm{~m}, 2 \mathrm{~F}, \mathrm{~F}^{12 / 13}\right),-161.2\left(\mathrm{~m}, 1 \mathrm{~F}, \mathrm{~F}^{14}\right),-163.9\left(\mathrm{~m}, 2 \mathrm{~F}, \mathrm{~F}^{12 / 13}\right)$.

HR-MS (FAB+):

calculated for $\mathrm{C}_{30} \mathrm{H}_{22} \mathrm{~F}_{5} \mathrm{~N}_{3}{ }^{58} \mathrm{NiO}_{2}$ :

$m / z=609.0986$

found

$m / z=609.1052$

Elemental analysis (\%):

calculated for $\mathrm{C}_{30} \mathrm{H}_{22} \mathrm{~F}_{5} \mathrm{~N}_{3} \mathrm{NiO}_{2}$ :

C 59.05, H 3.63, N 6.89

found:

C 59.05, H 3.78, N 6.96 


\section{Preparation of the $p$-fluorphenyl complex $\operatorname{LigNiC}_{6} \mathrm{H}_{4} \mathrm{~F}(4-\mathrm{F})$}

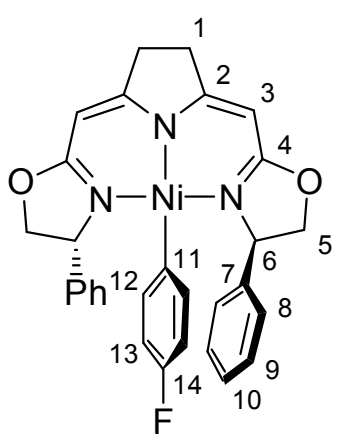

To a solution of the chlorido complex $2(192 \mathrm{mg}, 0.40 \mathrm{mmol})$ in $20 \mathrm{ml} \mathrm{THF}$, $p$-fluorophenylmagnesium bromide $(0.48 \mathrm{mmol}, 1.2 \mathrm{eq})$ was added at $0{ }^{\circ} \mathrm{C}$. After 10 min the cooling bath was removed and the reaction was stirred for another $5 \mathrm{~min}$. After removal of the solvents, the residue was treated with a toluene/pentane mixture (1/15) and the solution was filtrated. After removal of the solvents, the crude was recrystallized from a toluene/pentane mixture at $-40{ }^{\circ} \mathrm{C}$ to give a yellow crystalline solid in $77 \%$ yield $(165 \mathrm{mg})$.

\section{${ }^{1} \mathrm{H}-\mathrm{NMR}\left(\mathrm{C}_{6} \mathrm{D}_{6}, 600.130 \mathrm{MHz}, 295 \mathrm{~K}\right)$ :}

$\delta(\mathrm{ppm})=7.10\left(\mathrm{dd},{ }^{3} J=8.2 \mathrm{~Hz},{ }^{3} J_{F H}=6.8 \mathrm{~Hz} 2 \mathrm{H}, \mathrm{H}^{12}\right), 7.08-7.00\left(\mathrm{~m}, 6 \mathrm{H}, \mathrm{H}^{9,10}\right), 6.79\left(\mathrm{~d},{ }^{3} J=6.7\right.$ $\left.\mathrm{Hz}, 4 \mathrm{H}, \mathrm{H}^{8}\right), 6.54\left(\mathrm{dd},{ }^{3} J=8.2 \mathrm{~Hz},{ }^{4} J_{F H}=9.9 \mathrm{~Hz}, 2 \mathrm{H}, \mathrm{H}^{13}\right), 5.17\left(\mathrm{~s}, 2 \mathrm{H}, \mathrm{H}^{3}\right), 3.84\left(\mathrm{dd},{ }^{3} J=8.5 \mathrm{~Hz},{ }^{3} J\right.$ $\left.=2.0 \mathrm{~Hz}, 2 \mathrm{H}, \mathrm{H}^{6}\right), 3.55\left(\mathrm{dd},{ }^{3} J=8.5 \mathrm{~Hz},{ }^{2} J=8.1 \mathrm{~Hz}, 2 \mathrm{H}, \mathrm{H}^{5}\right), 3.43\left(\mathrm{dd},{ }^{2} J=8.1 \mathrm{~Hz},{ }^{3} J=2.3 \mathrm{~Hz}, 2 \mathrm{H}\right.$, $\left.\mathrm{H}^{5 *}\right), 2.34-2.10\left(\mathrm{~m}, 4 \mathrm{H}, \mathrm{H}^{1,1^{\circ}}\right)$.

${ }^{13} \mathrm{C}-\mathrm{NMR}\left(\mathrm{C}_{6} \mathrm{D}_{6}, 150.903 \mathrm{MHz}, 295 \mathrm{~K}\right)$ :

$\delta(\mathrm{ppm})=171.5\left(\mathrm{C}^{2}\right), 166.1\left(\mathrm{C}^{4}\right), 161.6\left(\mathrm{~d},{ }^{1} \mathrm{~J}_{\mathrm{CF}}=238.6 \mathrm{~Hz}, \mathrm{C}^{14}\right), 149.7\left(\mathrm{~d},{ }^{4} \mathrm{~J}_{\mathrm{CF}}=2.9 \mathrm{~Hz}, \mathrm{C}^{11}\right), 144.4$ $\left(\mathrm{C}^{7}\right), 137.5\left(\mathrm{~d},{ }^{2} \mathrm{~J}_{\mathrm{CF}}=5.0 \mathrm{~Hz}, \mathrm{C}^{12}\right), 128.4\left(\mathrm{C}^{9}\right), 127.3\left(\mathrm{C}^{10}\right), 126.1\left(\mathrm{C}^{8}\right), 111.8\left(\mathrm{~d},{ }^{3} \mathrm{~J}_{\mathrm{CF}}=17.8 \mathrm{~Hz}, \mathrm{C}^{13}\right)$, $81.3\left(C^{3}\right), 73.4\left(C^{5}\right), 69.3\left(C^{6}\right), 31.0\left(C^{1}\right)$.

${ }^{19}$ F-NMR $\left(\mathrm{C}_{6} \mathrm{D}_{6}, 376.273 \mathrm{MHz}, 295 \mathrm{~K}\right)$ :

$\delta(\mathrm{ppm})=123.9(\mathrm{~m})$

\section{HR-MS (FAB+):}

calculated for $\mathrm{C}_{30} \mathrm{H}_{26} \mathrm{FN}_{3}{ }^{58} \mathrm{NiO}_{2}$ :

$m / z=537.1363$

found

$m / z=537.1350$

\section{Elemental analysis (\%):}

calculated for $\mathrm{C}_{30} \mathrm{H}_{26} \mathrm{FN}_{3} \mathrm{NiO}_{2}$ :

C 66.94, H 4.87, N 7.81

found:

C 66.63, H 4.53, N 7.68 
Preparation of the phenyl complex LigNiPh (4-H)

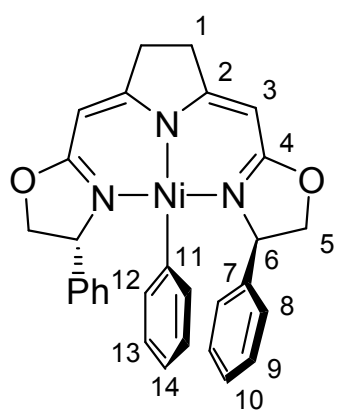

To a solution of the chlorido complex $2(113 \mathrm{mg}, 0.24 \mathrm{mmol})$ in $5 \mathrm{ml} \mathrm{THF}$, $\mathrm{PhMgCl}(0.31 \mathrm{mmol}, 1.3 \mathrm{eq})$ was added at $0{ }^{\circ} \mathrm{C}$. After $5 \mathrm{~min}$ the cooling bath was removed and the reaction was stirred for another $5 \mathrm{~min}$. After removal of the solvents, the residue was treated with a toluene/pentane mixture $(1 / 20)$ and the solution was filtrated. After removal of the solvents the crude was recrystallized from a toluene/pentane mixture $(1 / 30)$ at $-40{ }^{\circ} \mathrm{C}$ to give a yellow crystalline solid in $41 \%$ yield $(50 \mathrm{mg})$.

\section{${ }^{1} \mathrm{H}-\mathrm{NMR}\left(\mathrm{C}_{6} \mathrm{D}_{6}, 600.130 \mathrm{MHz}, 295 \mathrm{~K}\right)$ :}

$\delta(\mathrm{ppm})=7.35\left(\mathrm{~m}, 2 \mathrm{H}, \mathrm{H}^{12}\right), 7.11-7.01\left(\mathrm{~m}, 6 \mathrm{H}, \mathrm{H}^{9,10}\right), 6.90-6.84\left(\mathrm{~m}, 5 \mathrm{H}, \mathrm{H}^{8,14}\right), 6.74\left(\mathrm{~m}, 2 \mathrm{H}, \mathrm{H}^{13}\right)$, $5.19\left(\mathrm{~s}, 2 \mathrm{H}, \mathrm{H}^{3}\right), 4.00\left(\mathrm{dd},{ }^{3} J=8.6 \mathrm{~Hz},{ }^{3} J=2.1 \mathrm{~Hz}, 2 \mathrm{H}, \mathrm{H}^{6}\right), 3.56\left(\mathrm{dd},{ }^{3} J=8.6 \mathrm{~Hz},{ }^{2} J=8.0 \mathrm{~Hz}, 2 \mathrm{H}\right.$, $\left.\mathrm{H}^{5}\right), 3.45\left(\mathrm{dd},{ }^{2} \mathrm{~J}=8.0 \mathrm{~Hz},{ }^{3} \mathrm{~J}=2.2 \mathrm{~Hz}, 2 \mathrm{H}, \mathrm{H}^{5^{\circ}}\right), 2.18\left(\mathrm{~m}, 4 \mathrm{H}, \mathrm{H}^{1,1^{\circ}}\right)$.

${ }^{13} \mathrm{C}-\mathrm{NMR}\left(\mathrm{C}_{6} \mathrm{D}_{6}, 150.903 \mathrm{MHz}, 295 \mathrm{~K}\right)$ :

$\delta(\mathrm{ppm})=171.4\left(\mathrm{C}^{2}\right), 166.1\left(\mathrm{C}^{4}\right), 158.9\left(\mathrm{C}^{14}\right), 144.7\left(\mathrm{C}^{7}\right), 137.8\left(\mathrm{C}^{12}\right), 128.4\left(\mathrm{C}^{9}\right), 127.2\left(\mathrm{C}^{10}\right), 126.2$ $\left(C^{8}\right), 125.1\left(C^{13}\right), 122.4\left(C^{11}\right), 81.3\left(C^{3}\right), 73.4\left(C^{5}\right), 69.4\left(C^{6}\right), 54.8\left(C^{15}\right), 31.0\left(C^{1}\right)$.

\section{HR-MS (FAB+):}

calculated for $\mathrm{C}_{30} \mathrm{H}_{27} \mathrm{~N}_{3}{ }^{58} \mathrm{NiO}_{2}$ :

$m / z=519.1457$

found

$m / z=519.1465$

\section{Elemental analysis (\%):}

calculated for $\mathrm{C}_{30} \mathrm{H}_{27} \mathrm{~N}_{3} \mathrm{NiO}_{2}$ :

C 69.26, H 5.23, N 8.08

found:

C 69.09, H 5.24, N 7.94 


\section{Preparation of the $p$-methoxyphenyl complex $\mathrm{LigNiC}_{6} \mathrm{H}_{4} \mathrm{OMe}$ (4-OMe)}

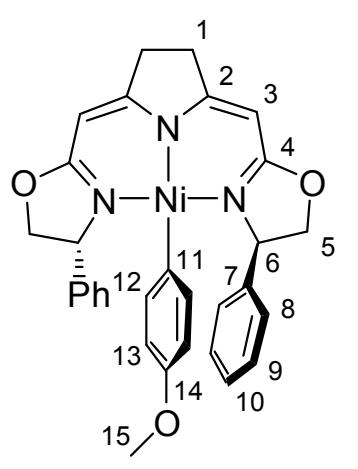

To a solution of the chlorido complex $2(100 \mathrm{mg}, 0.21 \mathrm{mmol})$ in $10 \mathrm{ml} \mathrm{Et}_{2} \mathrm{O}$ and $5 \mathrm{ml}$ toluene, $p$-methoxyphenylmagnesium bromide $(0.25 \mathrm{mmol}, 1.2 \mathrm{eq})$ was added at $-78{ }^{\circ} \mathrm{C}$. After 5 min the cooling bath was removed and the reaction was stirred for another $30 \mathrm{~min}$. After removal of the solvents, the residue was treated with a toluene/pentane mixture (1/6) and the solution was filtrated. After removal of the solvents, the crude was recrystallized from a toluene $/ \mathrm{Et}_{2} \mathrm{O} /$ pentane mixture at $-40{ }^{\circ} \mathrm{C}$ to give a yellow crystalline solid in $88 \%$ yield (101 mg).

\section{${ }^{1} \mathrm{H}-\mathrm{NMR}\left(\mathrm{C}_{6} \mathrm{D}_{6}, 600.130 \mathrm{MHz}, 295 \mathrm{~K}\right)$ :}

$\delta(\mathrm{ppm})=7.15\left(\mathrm{~d},{ }^{3} J=8.6 \mathrm{~Hz}, 2 \mathrm{H}, \mathrm{H}^{12}\right), 7.10-7.05\left(\mathrm{~m}, 4 \mathrm{H}, \mathrm{H}^{9}\right), 7.05-7.02\left(\mathrm{~m}, 2 \mathrm{H}, \mathrm{H}^{10}\right), 6.88\left(\mathrm{~d},{ }^{3} J=\right.$ $\left.7.2 \mathrm{~Hz}, 4 \mathrm{H}, \mathrm{H}^{8}\right), 6.49\left(\mathrm{~d},{ }^{3} J=8.6 \mathrm{~Hz}, 2 \mathrm{H}, \mathrm{H}^{13}\right), 5.20\left(\mathrm{~s}, 2 \mathrm{H}, \mathrm{H}^{3}\right), 4.04\left(\mathrm{dd},{ }^{3} J=8.5 \mathrm{~Hz},{ }^{3} J=2.1 \mathrm{~Hz}, 2\right.$ $\left.\mathrm{H}, \mathrm{H}^{6}\right), 3.60\left(\mathrm{dd},{ }^{2} J=8.1 \mathrm{~Hz},{ }^{3} J=8.5 \mathrm{~Hz}, 2 \mathrm{H}, \mathrm{H}^{5}\right), 3.49\left(\mathrm{~s}, 3 \mathrm{H}, \mathrm{H}^{15}\right), 3.47\left(\mathrm{dd},{ }^{2} J=8.1 \mathrm{~Hz},{ }^{3} J=2.1\right.$ $\left.\mathrm{Hz}, 2 \mathrm{H}, \mathrm{H}^{5^{\circ}}\right), 2.26-2.12\left(\mathrm{~m}, 4 \mathrm{H}, \mathrm{H}^{1,1^{\circ}}\right)$.

\section{${ }^{13} \mathrm{C}-\mathrm{NMR}\left(\mathrm{C}_{6} \mathrm{D}_{6}, 150.903 \mathrm{MHz}, 295 \mathrm{~K}\right)$ :}

$\delta(\mathrm{ppm})=171.4\left(\mathrm{C}^{2}\right), 165.0\left(\mathrm{C}^{4}\right), 157.2\left(\mathrm{C}^{14}\right), 144.7\left(\mathrm{C}^{7}\right), 144.6\left(\mathrm{C}^{11}\right), 137.3\left(\mathrm{C}^{12}\right), 128.3\left(\mathrm{C}^{9}\right), 127.1$ $\left(\mathrm{C}^{10}\right), 126.2\left(\mathrm{C}^{8}\right), 111.7\left(\mathrm{C}^{13}\right), 81.3\left(\mathrm{C}^{3}\right), 73.4\left(\mathrm{C}^{5}\right), 69.4\left(\mathrm{C}^{6}\right), 54.8\left(\mathrm{C}^{15}\right), 31.0\left(\mathrm{C}^{1}\right)$.

\section{HR-MS (FAB+):}

calculated for $\mathrm{C}_{31} \mathrm{H}_{29} \mathrm{~N}_{3}{ }^{58} \mathrm{NiO}_{2}$ :

found

\section{Elemental analysis (\%):}

calculated for $\mathrm{C}_{31} \mathrm{H}_{29} \mathrm{~N}_{3} \mathrm{NiO}_{2}$ :

found: $m / z=549.1562$

$m / z=549.1541$

C 67.66, H 5.31, N 7.64

C 67.68, H 5.20, N 7.65 


\section{Preparation of the dinuclear complex 4,4'-[LigNi $]_{2}$ Biphenyl (7)}

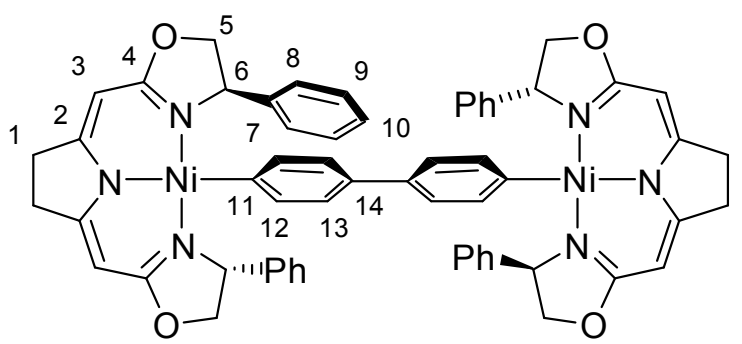

To a solution of 4,4'-dibromobiphenyl (15 mg, $0.048 \mathrm{mmol}$ ) and $3 \mathrm{ml} \mathrm{THF}$, a solution of the nickel(I) complex 1 ( $0.202 \mathrm{mmol}, 4.2 \mathrm{eq}$ in $2 \mathrm{ml}$ THF) was added at room temperature. After $1 \mathrm{~h}$ $\mathrm{LiEt}_{3} \mathrm{BH}(0.1 \mathrm{mmol}, 2.1 \mathrm{eq})$ was added and the solution was concentrated and stirred at $60{ }^{\circ} \mathrm{C}$ for 30 min to convert the generated nickel hydrido species $\mathbf{6}$ into the nickel(I) complex $\mathbf{1}$. Additional

4,4'-dibromobiphenyl ( $6.4 \mathrm{mg}, 0.02 \mathrm{mmol}, 0.42 \mathrm{eq}$.) was added and the reaction mixture was stirred at room temperature for $30 \mathrm{~min}$. Again, $\mathrm{LiEt}_{3} \mathrm{BH}$ was added $(0.07 \mathrm{mmol})$ and the solvents were removed. The residue was treated with a toluene/pentane mixture (2/1) and the solution was filtrated. After removal of the solvents, the residue was dissolved in toluene and the product was precipitated with pentane at $-40{ }^{\circ} \mathrm{C}$ to give a yellow solid in $50 \%$ yield $(52 \mathrm{mg})$.

${ }^{1} \mathrm{H}-\mathrm{NMR}\left(\mathrm{C}_{6} \mathrm{D}_{6}, 600.130 \mathrm{MHz}, 295 \mathrm{~K}\right)$ :

$\delta(\mathrm{ppm})=7.44\left(\mathrm{~d},{ }^{3} J=7.7 \mathrm{~Hz}, 4 \mathrm{H}, \mathrm{H}^{12}\right), 7.21\left(\mathrm{~d},{ }^{3} J=7.6 \mathrm{~Hz}, 4 \mathrm{H}, \mathrm{H}^{13}\right), 7.11-7.02\left(\mathrm{~m}, 12 \mathrm{H}, \mathrm{H}^{9,10}\right)$, $6.90\left(\mathrm{~d},{ }^{3} J=7.8 \mathrm{~Hz}, 8 \mathrm{H}, \mathrm{H}^{8}\right), 5.21\left(\mathrm{~s}, 4 \mathrm{H}, \mathrm{H}^{3}\right), 4.16\left(\mathrm{dd},{ }^{3} J=8.2 \mathrm{~Hz},{ }^{3} J=8.5 \mathrm{~Hz}, 4 \mathrm{H}, \mathrm{H}^{6}\right), 3.57$ (dd, $\left.{ }^{2} J=8.4 \mathrm{~Hz},{ }^{3} J=8.4 \mathrm{~Hz}, 4 \mathrm{H}, \mathrm{H}^{5}\right), 3.43\left(\mathrm{dd},{ }^{2} J=8.2 \mathrm{~Hz},{ }^{3} J=2.3 \mathrm{~Hz}, 4 \mathrm{H}, \mathrm{H}^{5^{\circ}}\right), 2.19\left(\mathrm{~m}, 8 \mathrm{H}, \mathrm{H}^{1{ }^{\circ}}\right)$.

\section{${ }^{13} \mathrm{C}-\mathrm{NMR}\left(\mathrm{C}_{6} \mathrm{D}_{6}, 150.903 \mathrm{MHz}, 295 \mathrm{~K}\right)$ :}

$\delta(\mathrm{ppm})=171.6\left(\mathrm{C}^{2}\right), 166.0\left(\mathrm{C}^{4}\right), 156.5\left(\mathrm{C}^{14}\right), 144.7\left(\mathrm{~d}, \mathrm{C}^{7}\right), 138.0\left(\mathrm{C}^{12}\right), 136.5\left(\mathrm{C}^{11}\right), 128.4\left(\mathrm{C}^{9}\right), 127.2$ $\left(\mathrm{C}^{10}\right), 126.3\left(\mathrm{C}^{8}\right), 123.2\left(\mathrm{C}^{13}\right), 81.3\left(\mathrm{C}^{3}\right), 73.4\left(\mathrm{C}^{5}\right), 69.4\left(\mathrm{C}^{6}\right), 31.0\left(\mathrm{C}^{1}\right)$.

\section{HR-MS (FAB+):}

calculated for $\mathrm{C}_{60} \mathrm{H}_{52} \mathrm{~N}_{6}{ }^{58} \mathrm{Ni}_{2} \mathrm{O}_{4}$ :

found

Elemental analysis (\%):

calculated for $\mathrm{C}_{60} \mathrm{H}_{52} \mathrm{~N}_{6} \mathrm{Ni}_{2} \mathrm{O}_{4}$ :

found: $m / z=1036.2757$

$m / z=1036.2710$

C 69.39, H 5.05, N 8.09

C 68.92, H 5.03, N 7.98 


\section{Preparation of the tetranuclear 4,7,11,14-[LigNi $]_{4}$ TAPP (8)}

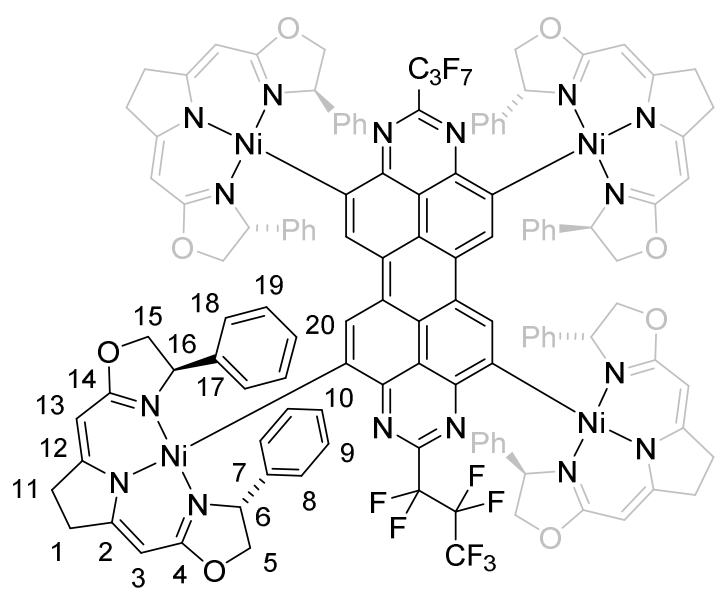

To a solution of 9-Bisperfluoropropyl 4,7,11,14tetrabromo-1,3,8,10-tetraazaperopyren $\quad(29 \mathrm{mg}$, $0.030 \mathrm{mmol}$ ) in $30 \mathrm{ml} \mathrm{THF}$, a solution of the nickel(I) complex 1 (132 mg, $0.298 \mathrm{mmol}, 8.9 \mathrm{eq}$ ) was added at $0{ }^{\circ} \mathrm{C}$ and stirred for $10 \mathrm{~h}$. After removal of the solvents, the residue was treated with toluene, stirred and pentane was added to precipitate the product. After filtration, the crude was recrystallized from a $\mathrm{THF} / \mathrm{Et}_{2} \mathrm{O} /$ pentane mixture at room temperature to give a red crystalline solid in $38 \%$ yield $(28 \mathrm{mg})$.

\section{${ }^{1} \mathrm{H}-\mathrm{NMR}\left(\mathrm{C}_{6} \mathrm{D}_{6}, 600.130 \mathrm{MHz}, 295 \mathrm{~K}\right)$ :}

$\delta(\mathrm{ppm})=9.44\left(\mathrm{~s}, 4 \mathrm{H}, \mathrm{H}^{\mathrm{TAPP}}\right), 7.83\left(\mathrm{~d},{ }^{3} J=7.5 \mathrm{~Hz}, 8 \mathrm{H}, \mathrm{H}^{8}\right), 7.58\left(\mathrm{dd},{ }^{3} J=7.4 \mathrm{~Hz},{ }^{3} J=7.3, \mathrm{~Hz} 8 \mathrm{H}\right.$, $\left.\mathrm{H}^{9}\right), 7.42\left(\mathrm{t},{ }^{3} J=7.3 \mathrm{~Hz}, 4 \mathrm{H}, \mathrm{H}^{10}\right), 6.49\left(\mathrm{t},{ }^{3} J=7.2 \mathrm{~Hz}, 4 \mathrm{H}, \mathrm{H}^{20}\right), 6.37\left(\mathrm{dd},{ }^{3} J=7.4 \mathrm{~Hz},{ }^{3} J=7.3 \mathrm{~Hz}, 8\right.$ $\left.\mathrm{H}, \mathrm{H}^{19}\right), 5.72\left(\mathrm{~d},{ }^{3} J=7.4 \mathrm{~Hz}, 8 \mathrm{H}, \mathrm{H}^{18}\right), 5.39\left(\mathrm{~s}, 4 \mathrm{H}, \mathrm{H}^{3 / 13}\right), 5.31\left(\mathrm{~s}, 4 \mathrm{H}, \mathrm{H}^{3 / 13}\right), 4.57\left(\mathrm{dd},{ }^{3} J=8.5 \mathrm{~Hz}\right.$, $\left.{ }^{3} J=2.5 \mathrm{~Hz}, 4 \mathrm{H}, \mathrm{H}^{6}\right), 4.18\left(\mathrm{dd},{ }^{2} J=8.2 \mathrm{~Hz},{ }^{3} J=8.6 \mathrm{~Hz}, 4 \mathrm{H}, \mathrm{H}^{5}\right), 3.87\left(\mathrm{dd},{ }^{2} J=8.2 \mathrm{~Hz},{ }^{3} J=8.6 \mathrm{~Hz}, 4\right.$ $\left.\mathrm{H}, \mathrm{H}^{5}\right), 3.60\left(\mathrm{dd},{ }^{3} J=8.0 \mathrm{~Hz},{ }^{3} J=1.6 \mathrm{~Hz}, 4 \mathrm{H}, \mathrm{H}^{16}\right), 3.45\left(\mathrm{dd},{ }^{2} J=8.2 \mathrm{~Hz},{ }^{3} J=8.3 \mathrm{~Hz}, 4 \mathrm{H}, \mathrm{H}^{15}\right), 2.98$ $\left(\mathrm{dd},{ }^{2} J=8.0 \mathrm{~Hz},{ }^{3} J=1.9 \mathrm{~Hz}, 4 \mathrm{H}, \mathrm{H}^{15^{\circ}}\right), 2.53-2.29\left(\mathrm{~m}, 16 \mathrm{H}, \mathrm{H}^{1,1^{\circ}, 11,11^{\circ}}\right)$.

\section{${ }^{13} \mathrm{C}-\mathrm{NMR}\left(\mathrm{C}_{6} \mathrm{D}_{6}, 150.903 \mathrm{MHz}, 295 \mathrm{~K}\right)$ :}

$\delta(\mathrm{ppm})=172.5\left(\mathrm{C}^{2 / 1212}\right), 172.4\left(\mathrm{C}^{2 / 12}\right), 166.1\left(\mathrm{C}^{14}\right), 165.7\left(\mathrm{C}^{4}\right), 160.7\left(\mathrm{C}^{\mathrm{TAPP}}\right), 160.4\left(\mathrm{C}^{\mathrm{TAPP}}\right), 144.9$ $\left(\mathrm{C}^{7}\right), 142.2\left(\mathrm{C}^{17}\right), 138.2\left(\mathrm{CH}^{\mathrm{TAPP}}\right), 129.4\left(\mathrm{C}^{9}\right), 127.8\left(\mathrm{C}^{10}\right), 127.6\left(\mathrm{C}^{\mathrm{TAPP}}\right), 127.3\left(\mathrm{C}^{19}\right), 126.7\left(\mathrm{C}^{8}\right), 126.4$ $\left(\mathrm{C}^{20}\right), 124.7\left(\mathrm{C}^{18}\right), 122.1\left(\mathrm{C}^{\mathrm{TAPP}}\right), 119.0\left(\mathrm{C}^{\mathrm{TAPP}}\right), 112.7\left(\mathrm{C}^{\mathrm{TAPP}}\right), 81.8\left(\mathrm{C}^{3 / 13}\right), 80.8\left(\mathrm{C}^{3 / 13}\right), 73.8\left(\mathrm{C}^{5}\right)$, $73.6\left(\mathrm{C}^{15}\right), 69.4\left(\mathrm{C}^{6,16}\right), 31.1\left(\mathrm{C}^{1,11}\right)$.

${ }^{19}$ F-NMR $\left(C_{6} D_{6}, 376.273 \mathrm{MHz}, 295 \mathrm{~K}\right)$ :

$\delta(\mathrm{ppm})=-79.7\left(\mathrm{t},{ }^{3} J=10.6 \mathrm{~Hz}, 6 \mathrm{~F}\right),-106.2-110.3(\mathrm{~m}, 4 \mathrm{~F}),-123.5(\mathrm{~m}, 4 \mathrm{H}) .(\mathrm{CR} 722-40010)$

\section{HR-MS (ESI+):}

calculated for $\mathrm{C}_{124} \mathrm{H}_{92} \mathrm{~F}_{14} \mathrm{~N}_{16}{ }^{58} \mathrm{Ni}_{4} \mathrm{O}_{8}: \quad \quad m / z=2430.4474$

found

$m / z=2430.4484$

\section{Elemental analysis (\%):}

calculated for $\mathrm{C}_{124} \mathrm{H}_{92} \mathrm{~F}_{14} \mathrm{~N}_{16} \mathrm{Ni}_{4} \mathrm{O}_{8}: \quad$ C 61.17, H 3.81, N 9.20

found:

C 61.38, H 4.17, N 9.30 


\section{S1.3 Additional Experiments}

Catalytic hydrodehalogenation of $p$-chlorofluorobenzene (NMR experiment)

A NMR sample was prepared using $p$-chlorofluorobenzene (13.1 mg, $0.1 \mathrm{mmol}), \mathrm{LiEt}_{3} \mathrm{BH}$ (0.21 mmol), complex $2(4.8 \mathrm{mg}, 0.01 \mathrm{mmol}, 10 \mathrm{~mol} \%)$ and 1,4-bis(trifluoromethyl)benzene as internal standard in $0.5 \mathrm{ml}$ THF- $\mathrm{d}_{8}$ and the reaction was monitored by ${ }^{19} \mathrm{~F}$ NMR spectroscopy.

\section{Initial rate determination of the reaction of the nickel(I) complex 1 with $p$-chlorofluorobenzene}

The order of the reaction of $\mathbf{1}$ with $p$-chlorofluorobenzene was determined by initial rate measurements in a series of kinetic experiments using ${ }^{19} \mathrm{~F} \mathrm{NMR}$ spectroscopy. Therefore, the concentration of $\mathbf{1}$ and later of $p$-chlorofluorobenzene was varied in two series of NMR measurements at $295 \mathrm{~K}$ in $\mathrm{C}_{6} \mathrm{D}_{6}$.

In the first series, a solution of $p$-chlorofluorobenzene $(5 \mathrm{mg})$ and 1,4-dimethoxybenzene as internal standard in $0.2 \mathrm{ml} \mathrm{C}_{6} \mathrm{D}_{6}$ was added to a solution of the nickel(I) complex 1 (1.25 mg, $2.5 \mathrm{mg}, 5.0 \mathrm{mg}$, $7.5 \mathrm{mg}, 10.0 \mathrm{mg}, 12.5 \mathrm{mg}, 15.0 \mathrm{mg}$ ) in $0.4 \mathrm{ml} \mathrm{C}_{6} \mathrm{D}_{6}$, respectively and the reaction was monitored by

${ }^{19} \mathrm{~F}$ NMR spectroscopy. In a second series, a solution of nickel(I) complex 1 (5.0 mg) in $0.2 \mathrm{ml} \mathrm{C}_{6} \mathrm{D}_{6}$ was added to a solution of $p$-chlorofluorobenzene $(2.5 \mathrm{mg}, 5 \mathrm{mg}, 7.5 \mathrm{mg}, 10 \mathrm{mg}, 12.5 \mathrm{mg}, 15 \mathrm{mg})$ and 1,4-dimethoxybenzene in $0.4 \mathrm{ml} \mathrm{C}_{6} \mathrm{D}_{6}$, respectively. Additionally, the temperature dependency of the initial rate was probed using $5 \mathrm{mg} 1$ and $5 \mathrm{mg} p$-chlorofluorobenzene $\left(0.6 \mathrm{ml} \mathrm{C}_{6} \mathrm{D}_{6}\right)$ at $283 \mathrm{~K}, 303 \mathrm{~K}$, $313 \mathrm{~K}, 333 \mathrm{~K}$.

The initial rate of each run was determined by fitting (least squares, no constrains) the general function of a second-order reaction (1) to the data obtained from the NMR measurements (intensity of the ${ }^{19} \mathrm{~F}$ NMR signal corresponding to the aryl complex 4-F vs. time). The value of the first derivative of the fitted function at $\mathrm{y}=0$ was used as the initial rate which were plotted against the concentration of $\mathbf{1}$ or $p$-chlorofluorobenzene, respectively.

$$
a-(a(a-b) \exp (k(a-b)(x-c)) /(a \times \exp (k(a-b)(x-c))-b))
$$

\section{Determining the relative rates of the reaction of differently substituted chlorobenzenes with the} nickel(I) complex-Hammett plot

The relative rates of the reaction of $p$-substituted chlorobenzenes $\left(\mathrm{R}=\mathrm{MeO}, \mathrm{CH}_{3}, \mathrm{H}, \mathrm{F}, \mathrm{CF}_{3}\right)$ with the nickel(I) complex 1 under pseudo-first-order-conditions was determined in three speperately performed competition experiments (A: $\mathrm{F}$ and $\mathrm{MeO} ; \mathrm{B}: \mathrm{F}, \mathrm{H}$ and $\mathrm{CH}_{3} ; \mathrm{C}: \mathrm{F}$ and $\mathrm{CF}_{3}$ ). Therefore, an excess of the corresponding $p$-chlorobenzenes (each: $0.339 \mathrm{mmol}, 10 \mathrm{eq}$ ) was dissolved in $12 \mathrm{ml}$ THF and held at $295 \mathrm{~K}$. A solution of the nickel(I) complex $(15.0 \mathrm{mg}, 0.034 \mathrm{mmol})$ in $2 \mathrm{ml}$ THF was added and the reaction mixture was stirred for $16 \mathrm{~h}$. The solvent was removed and the residue was analyzed by NMR spectroscopy in $\mathrm{C}_{6} \mathrm{D}_{6}$. The ratio of the integrals of the $\mathrm{H}^{6}$ proton signals (for $\mathrm{R}=\mathrm{MeO}, \mathrm{H}, \mathrm{F}$ see above; the complexes with $\mathrm{R}=\mathrm{Me}\left(\mathrm{H}^{6}=4.07 \mathrm{ppm}\right)$ and $\mathrm{CF}_{3}\left(\mathrm{H}^{6}=3.75 \mathrm{ppm}\right)$ were not separately prepared and isolated) were used for a Hammett plot (Figure 3).

\section{EPR analysis of ongoing catalytic hydrodehalogenation of 2,2-dichlorotetrahydronaphthalene}

A solution was prepared at $-40{ }^{\circ} \mathrm{C}$ using $15 \mathrm{mg}$ 2,2-dichlorotetrahydronaphthalene, $4.6 \mathrm{mg}$ of the nickel(I) complex 1 and $0.2 \mathrm{mmol}$ of $\mathrm{LiEt}_{3} \mathrm{BH}$ in $0.5 \mathrm{ml}$ THF. A small amount of the solution was transferred into a EPR tube, the sample was allowed to warm to room temperature for $5 \mathrm{~min}$ and 
subsequently frozen using liquid $\mathrm{N}_{2}$. The analysis was carried out at $110 \mathrm{~K}, v=9.442171 \mathrm{GHz}$, power $19.97 \mathrm{~mW}$, power attenuation $10.0 \mathrm{~dB}$.

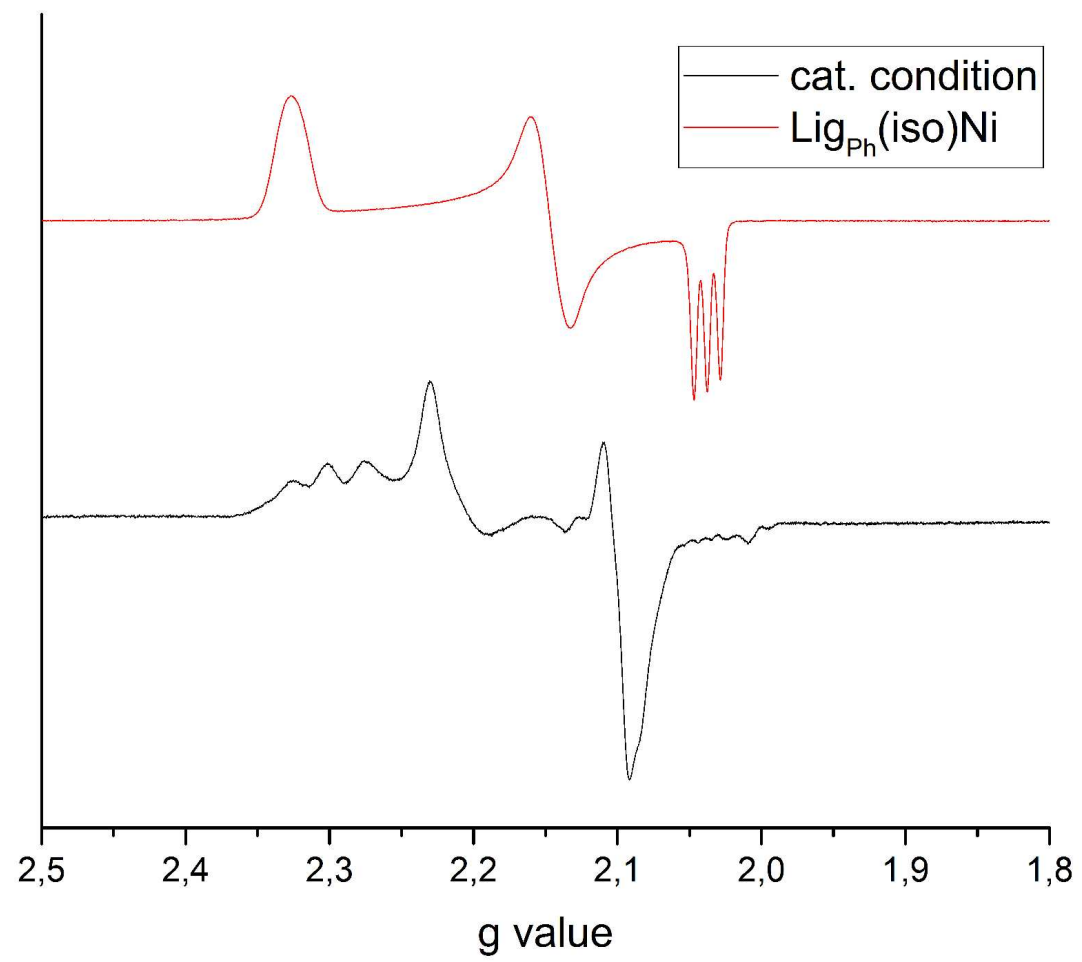

Figure 1. EPR spectrum of the ongoing catalytic hydrodehalogenation of 2,2dichlorotetrahydronaphthalene using the nickel(I) complex $\mathbf{1}$ as catalyst and LiEt3BH as hydride source in THF (bottom) and the EPR spectrum of the corresponding nickel(I) complex 1 (top).

\section{S2. Computational Data}

DFT calculations were performed using the Gaussian 09, Revision D.01 software package ${ }^{5}$ on the bwforcluster JUSTUS. The geometry optimization and the harmonic frequency analysis were carried out on the unrestricted B3LYP/6-311G(d,p) ${ }^{6}$ level of theory including Grimme D3 dispersion correction ${ }^{7}$ and SMD polarizable continuum model ${ }^{8}$ using the "tight" convergence criteria for SCF calculations. The connection between the transition states and minimum geometries was verified by two separate optimizations starting from the TS geometry with a slight distortion along either direction of the negative vibrational mode. 


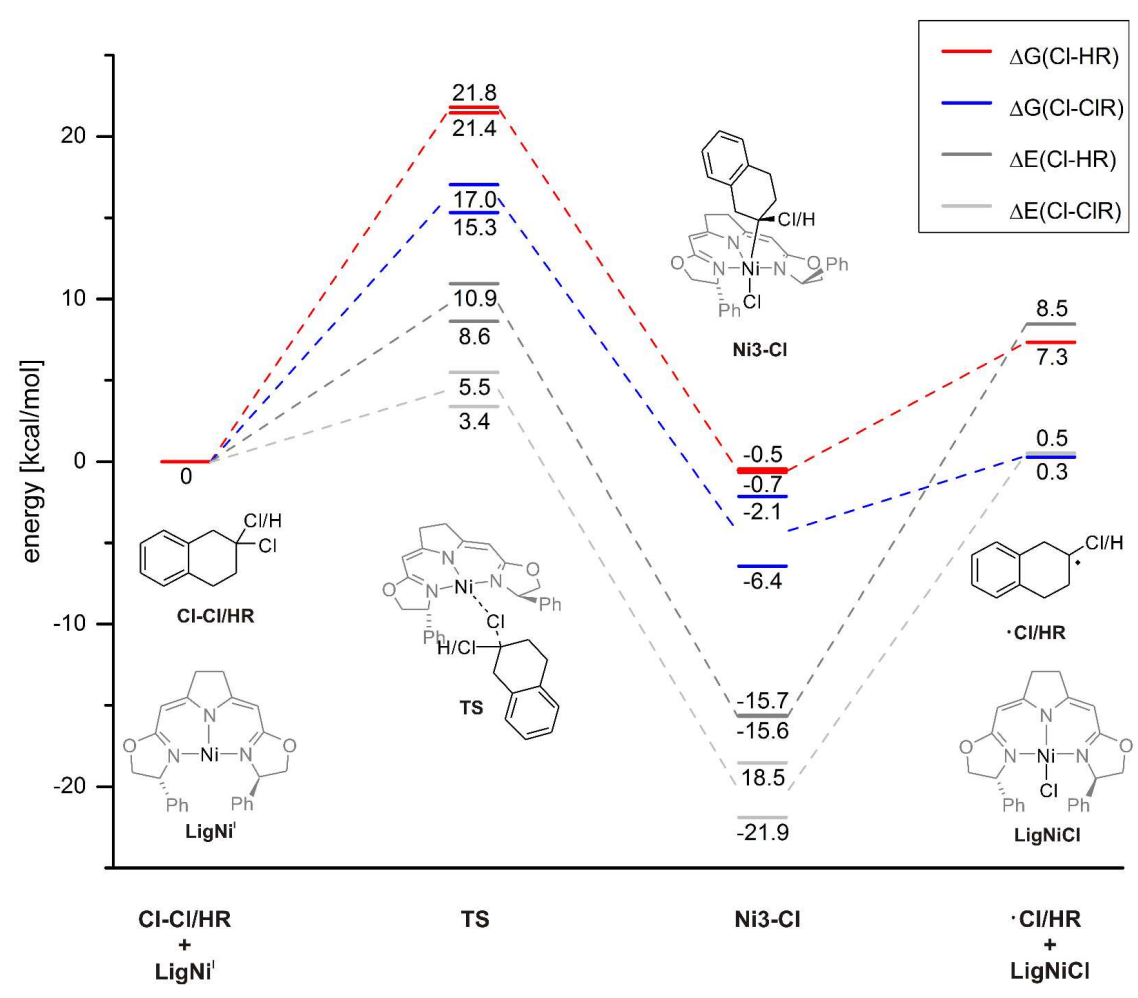

Figure 2. Energy profile (DFT) of both the activation of 2-chlorotetrahydronaphthalene (2 enantiomers) as well as 2,2-dichlorotetrahydronaphthalene by the nickel(I) complex $1 \Delta \mathrm{G}(\mathrm{Cl}-\mathrm{HR})$ and $[\Delta \mathrm{G}(\mathrm{Cl}-\mathrm{ClR})$, respectively. In the absence of the second chlorine atom in the case of the two 2chlorotetrahydronaphthalene enantiomers (Cl-HR) activation barriers of $21.8 \mathrm{kcal} / \mathrm{mol}$ and $21.4 \mathrm{kcal} / \mathrm{mol}$ were found, which are significantly higher than the two diasteriomeric TS of $17.0 \mathrm{kcal} / \mathrm{mol}$ and $15.3 \mathrm{kcal} / \mathrm{mol}$ for 2,2-dichlorotetrahydronaphthalene (Cl-ClR). The figure shows the key geometries of the processes as generalized illustrations in which either an $\mathrm{H}$ or $\mathrm{Cl}$ atom is located in alpha position to the activation site $(\mathrm{H} / \mathrm{ClR})$. 


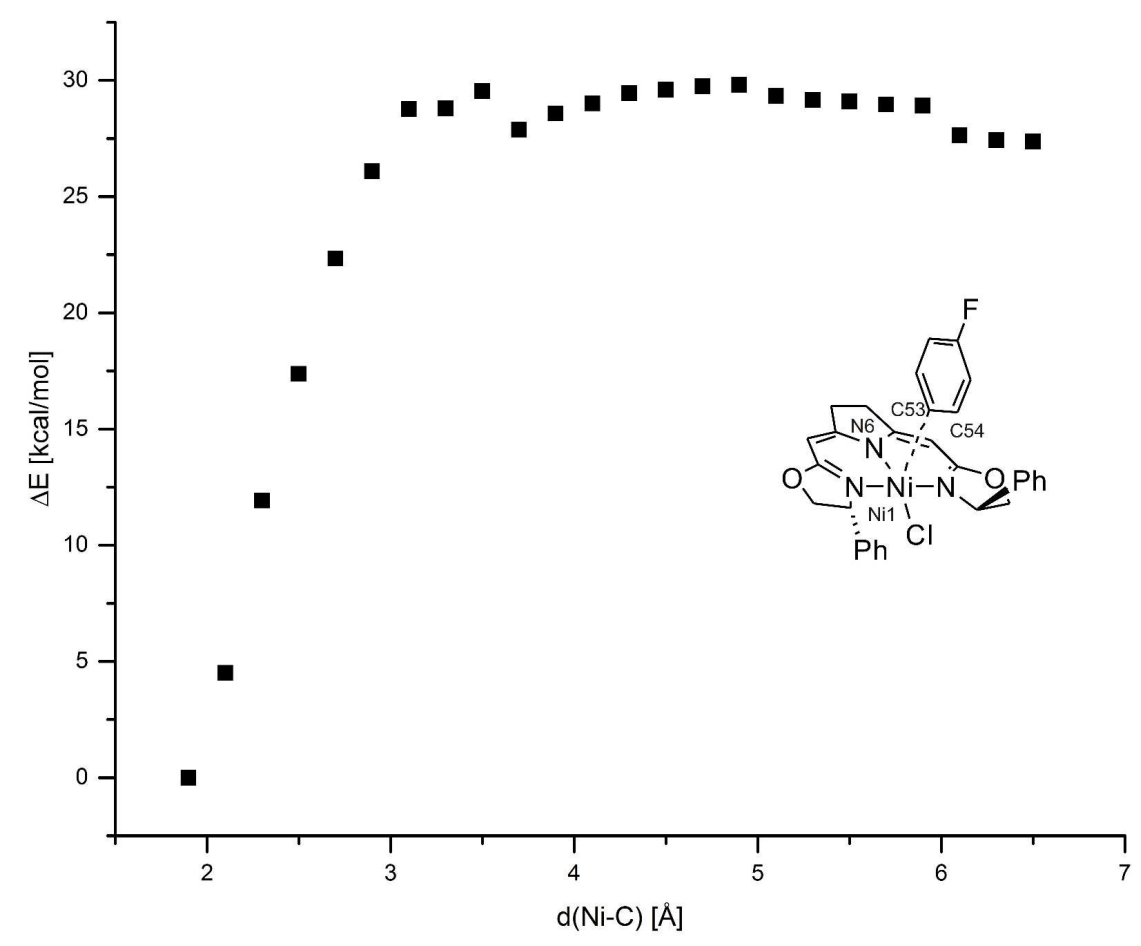

Figure 3. PES scan of the homolytic cleavage of the Ni-aryl bond for the nickel(III)aryl complex (Ni3_Cl_ $\mathbf{C}_{6} \mathbf{H}_{4} \mathbf{F}$ ) showing no energetic barrier. Due to difficulties with convergence the relaxed PES scan was restricted with the two angles N6-Ni1-C53 and Ni1-C53-C54 being frozen.

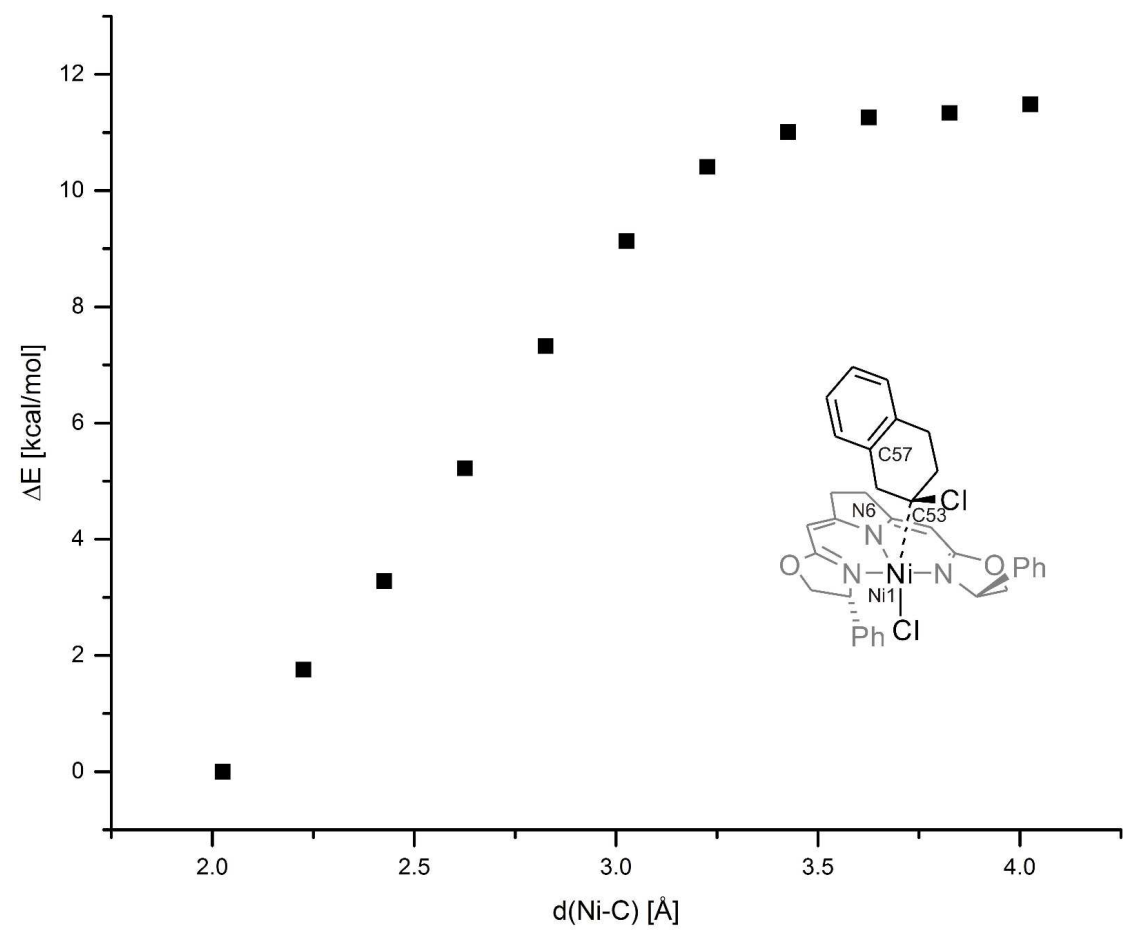

Figure 4. PES scan of the homolytic cleavage of the Ni-C bond for the nickel(III)alkyl complex (Ni3_Cl-dia_r) showing no energetic barrier. Due to difficulties with convergence the relaxed PES scan was restricted with the two angles N6-Ni1-C53 and Ni1-C53-C57 being frozen. 


\section{S3. Crystallographic Data}

Table S1. Details of the crystal structure determinations of 4-F, 4-H, 4-OMe and $8 \cdot x$ thf.

\begin{tabular}{|c|c|c|c|c|}
\hline & 4-F & $4-\mathrm{H}$ & 4-OMe & $8 \cdot x$ thf \\
\hline formula & $\mathrm{C}_{30} \mathrm{H}_{26} \mathrm{FN}_{3} \mathrm{NiO}_{2}$ & $\mathrm{C}_{30} \mathrm{H}_{27} \mathrm{~N}_{3} \mathrm{NiO}_{2}$ & $\mathrm{C}_{31} \mathrm{H}_{29} \mathrm{~N}_{3} \mathrm{NiO}_{3}$ & $\mathrm{C}_{124} \mathrm{H}_{92} \mathrm{~F}_{14} \mathrm{~N}_{16} \mathrm{Ni}_{4} \mathrm{O}_{8}$ \\
\hline crystal system & monoclinic & tetragonal & monoclinic & trigonal \\
\hline space group & $P 2_{1}$ & $P 4_{1}$ & $P 2_{1}$ & $P 3_{1} 21$ \\
\hline$a / \AA$ & $9.68844(6)$ & $14.52683(2)$ & 11.10582(16) & $32.5209(3)$ \\
\hline$b / \AA$ & $18.62780(9)$ & & $6.60254(10)$ & \\
\hline$c / \AA$ & $13.98526(8)$ & 71.29645(17) & $18.2048(3)$ & $47.2676(4)$ \\
\hline$\beta /{ }^{\circ}$ & $99.6952(6)$ & & $105.4535(15)$ & \\
\hline$V / \AA^{3}$ & $2487.93(2)$ & $15045.60(6)$ & $1286.63(3)$ & $43293.1(8)$ \\
\hline$Z$ & 4 & 24 & 2 & 12 \\
\hline$M_{\mathrm{r}}$ & 538.25 & 520.25 & 550.28 & 2434.97 \\
\hline$F_{000}$ & 1120 & 6528 & 576 & 15000 \\
\hline$d_{\mathrm{c}} / \mathrm{Mg} \cdot \mathrm{m}^{-3}$ & 1.437 & 1.378 & 1.420 & 1.121 \\
\hline
\end{tabular}




\begin{tabular}{|c|c|c|c|c|}
\hline & 4-F & 4-H & 4-OMe & $\mathbf{8} \cdot x$ thf \\
\hline$\mu / \mathrm{mm}^{-1}$ & 1.463 & 1.372 & 1.399 & 1.176 \\
\hline max., min. transmission factors & $0.914,0.791$ & $1.0000,0.8234$ & $0.960,0.815$ & $1.0000,0.5633$ \\
\hline X-radiation, $\lambda / \AA$ & \multicolumn{4}{|c|}{$\mathrm{Cu}-K \alpha, 1.54184$} \\
\hline data collect. temperat. /K & $115(1)$ & $110(1)$ & $110(1)$ & $120(1)$ \\
\hline$\theta$ range ${ }^{\circ}$ & 4.0 to 72.0 & 3.6 to 70.0 & 4.2 to 71.9 & 3.7 to 73.3 \\
\hline index ranges $h, k, l$ & $-11 \ldots 11,-22 \ldots 22,-17 \ldots 17$ & $-17 \ldots 17,-17 \ldots 17,-86 \ldots 86$ & $-13 \ldots 13,-8 \ldots 7,-22 \ldots 22$ & $-39 \ldots 39,-39 \ldots 39,-57 \ldots 57$ \\
\hline reflections measured & 88074 & 377171 & 13326 & 1277962 \\
\hline unique $\left[R_{\text {int }}\right]$ & $9702[0.0485]$ & $28351[0.0341]$ & $4662[0.0264]$ & $55838[0.1044]$ \\
\hline observed $[I \geq 2 \sigma(I)]$ & 9572 & 28097 & 4530 & 40494 \\
\hline data / restraints / parameters & $9702 / 1 / 667$ & $28351 / 1 / 1945$ & $4662 / 1 / 344$ & $55838 / 3156 / 2920$ \\
\hline GooF on $F^{2}$ & 1.027 & 1.107 & 1.033 & 0.956 \\
\hline$R$ indices $[F>4 \sigma(F)] R(F), w R\left(F^{2}\right)$ & $0.0250,0.0658$ & $0.0246,0.0582$ & $0.0263,0.0638$ & $0.0784,0.2055$ \\
\hline$R$ indices (all data) $R(F), w R\left(F^{2}\right)$ & $0.0254,0.0662$ & $0.0249,0.0584$ & $0.0274,0.0648$ & $0.0939,0.2213$ \\
\hline absolute structure parameter & $-0.021(9)$ & $-0.008(2)$ & $-0.031(14)$ & $0.012(5)$ \\
\hline largest residual peaks $/ \mathrm{e} \cdot \AA^{-3}$ & $0.230,-0.241$ & $0.256,-0.257$ & $0.232,-0.261$ & $1.732,-0.500$ \\
\hline
\end{tabular}




\section{References}

1. Armarego, W. L. F.; Chai, C. L. L., Purification of Laboratory Chemicals. Elsevier/ButterworthHeinemann: 2009.

2. Konrad, F.; Lloret Fillol, J.; Wadepohl, H.; Gade, L. H., Inorg. Chem. 2009, 48, 8523.

3. Rettenmeier, C.; Wadepohl, H.; Gade, L. H., Chem. Eur. J. 2014, 20, 9657.

4. Fulmer, G. R.; Miller, A. J. M.; Sherden, N. H.; Gottlieb, H. E.; Nudelman, A.; Stoltz, B. M.; Bercaw, J. E.; Goldberg, K. I., Organometallics 2010, 29, 2176.

5. Frisch, M. J.; Trucks, G. W.; Schlegel, H. B.; Scuseria, G. E.; Robb, M. A.; Cheeseman, J. R.; Scalmani, G.; Barone, V.; Mennucci, B.; Petersson, G. A.; Nakatsuji, H.; Caricato, M.; Li, X.; Hratchian, H. P.; Izmaylov, A. F.; Bloino, J.; Zheng, G.; Sonnenberg, J. L.; Hada, M.; Ehara, M.; Toyota, K.; Fukuda, R.; Hasegawa, J.; Ishida, M.; Nakajima, T.; Honda, Y.; Kitao, O.; Nakai, H.; Vreven, T.; J. A. Montgomery, J.; Peralta, J. E.; Ogliaro, F.; Bearpark, M.; Heyd, J. J.; Brothers, E.; Kudin, K. N.; Staroverov, V. N.; Keith, T.; Kobayashi, R.; Normand, J.; Raghavachari, K.; Rendell, A.; Burant, J. C.; Iyengar, S. S.; Tomasi, J.; Cossi, M.; Rega, N.; Millam, J. M.; Klene, M.; Knox, J. E.; Cross, J. B.; Bakken, V.; Adamo, C.; Jaramillo, J.; Gomperts, R.; Stratmann, R. E.; Yazyev, O.; Austin, A. J.; Cammi, R.; Pomelli, C.; Ochterski, J. W.; Martin, R. L.; Morokuma, K.; Zakrzewski, V. G.; Voth, G. A.; Salvador, P.; Dannenberg, J. J.; Dapprich, S.; Daniels, A. D.; Farkas, O.; Foresman, J. B.; Ortiz, J. V.; Cioslowski, J.; Fox, D. J. Gaussian 09, Revision D.01, Gaussian, Inc.: Wallingford CT, 2013.

6. (a) Wachters, A. J. H., J. Chem. Phys. 1970, 52, 1033; (b) Hay, P. J., J. Chem. Phys. 1977, 66, 4377; (c) Krishnan, R.; Binkley, J. S.; Seeger, R.; Pople, J. A., J. Chem. Phys. 1980, 72, 650; (d) McLean, A. D.; Chandler, G. S., J. Chem. Phys. 1980, 72, 5639; (e) Becke, A. D., Physical Review A 1988, 38, 3098; (f) Lee, C.; Yang, W.; Parr, R. G., Physical Review B 1988, 37, 785; (g) Miehlich, B.; Savin, A.; Stoll, H.; Preuss, H., Chem. Phys. Lett. 1989, 157, 200; (h) Raghavachari, K.; Trucks, G. W., J. Chem. Phys. 1989, 91, 1062; (i) Binning, R. C.; Curtiss, L. A., J. Comput. Chem. 1990, 11, 1206; (j) McGrath, M. P.; Radom, L., J. Chem. Phys. 1991, 94, 511; (k) Becke, A. D., J. Chem. Phys. 1993, 98, 5648; (I) Curtiss, L. A.; McGrath, M. P.; Blaudeau, J. P.; Davis, N. E.; Binning, R. C.; Radom, L., J. Chem. Phys. 1995, 103, 6104.

7. Grimme, S.; Antony, J.; Ehrlich, S.; Krieg, H., J. Chem. Phys. 2010, 132, 154104.

8. Marenich, A. V.; Cramer, C. J.; Truhlar, D. G., The Journal of Physical Chemistry B 2009, 113, 6378. 